\title{
Arthroscopic pie-crusting release of the posteromedial complex of the knee for surgical treatment of the medial meniscus injury
}

\section{Xu Han}

Henan Luoyang Orthopedic-Traumatological Hospital(Henan Orthopedic Hospital)

\section{Peizhao Wang}

Henan Luoyang Orthopedic-Traumatological Hospital(Henan Orthooedic Hospital)

Jinyang Yu

Henan Luoyang Orthopedic-Traumatological Hospital (Henan Orthopedic Hospital)

\section{Xiao Wang}

Henan Luoyang Orthopedic-Traumatological Hospital(Henan Orthopedic Hospital)

Honglue Tan ( $\nabla$ hnlc.love@163.com )

Luoyang Orthopedic-Traumatological Hospital (Henan Orthopedic Hospital)

Research article

Keywords: Posteromedial complex, Meniscus injury, Pie-crusting release, Arthroscopy, Knee

Posted Date: February 21st, 2020

DOI: https://doi.org/10.21203/rs.2.24229/v1

License: (c) (i) This work is licensed under a Creative Commons Attribution 4.0 International License. Read Full License 


\section{Abstract}

Background: Arthroscopic narrow posteromedial gap of the knee may cause the failure of meniscus operation. The posteromedial complex (PMC) of the knee, including the posterior part of MCL and posterior oblique ligament, has a restrictive effect on the opening of the posteromedial gap of the knee in the half-extension position. Thus, we evaluated the radiological and clinical results of pie-crusting release of PMC for arthroscopic meniscal surgery in tight knees.

Methods : Sixty patients with posterior injury of the medial meniscus were reviewed. All patients accepted arthroscopic pie-crusting release of the PMC. Fourty patients accepted meniscoplasty, and 20 patients accepted meniscuc suturing. To evaluate the arthroscopic opening of the medial gap in $20^{\circ}$ halfextension under $11-\mathrm{kg}$ valgus stress, the width of the medial space before and after release were measured. During follow-up, the medial stability was evaluated by radiographic measurements of the joint space width (JSW) in $20^{\circ}$ half-extension. MRI was conducted to evaluate the healing of MCL and meniscus. Knee functions were evaluated using VAS, Lysholm, IKDC and Tegner scoring systems.

Results: In all patients, meniscus operations were performed without iatrogenic cartilage injury. After PMC release, the arthroscopic width of the medial space was $5.7 \pm 0.5 \mathrm{~mm}$, showing larger than that before release $(2.5 \pm 0.5 \mathrm{~mm})(p<0.01)$. The follow-up time was $21.93 \pm 7.04$ months, there was no residual valgus laxity of the knee. The radiographic JSW was $5.97 \pm 0.8 \mathrm{~mm}$ preoperatively, $9.2 \pm 1.1 \mathrm{~mm}$ in the postoperative $1 \mathrm{st}$ week, and $6.1 \pm 0.9 \mathrm{~mm}$ in the postoperative 3rd months, showing no differences between pre- and postoperative 3rd month ( $p>0.05$ ). For sutured meniscus, MRI showed healing in 15 patients while five had two-grade abnormal signals. VAS, Lysholm, IKDC and Tegner scores were $1.80 \pm 0.51,80.08 \pm 3.74,82.17 \pm 4.64$ and $5.48 \pm 0.59$, respectively, showing significant differences compared with the preoperative scores $(5.57 \pm 0.69,48.17 \pm 4.22,51.42 \pm 4.02$ and $3.20 \pm 0.68$, respectively) $(P<0.01)$.

Conclusion s: Pie-crusting release of PMC can increase the posteromedial space and improve the visual field of the knee under arthroscopy, and this technique do neither produce residual valgus instability of the knee nor affect clinical outcome at the final follow-up.

\section{Background}

Medial meniscus injury is a common cause of knee joint pain. It is not properly treated for a long time, degenerative changes of the cartilage in the medial compartment of the knee joint will happen [1]. With the rapid development of arthroscopic minimally-invasive technology, this kind of injury involving the posterior part of the medial meniscus can be treated by means of microscopic repair or suture according to the different injury types, which ultimately avoid the occurrence or development of osteoarthritis [2]. However, during arthroscopic surgery, an unclear visual field of the posterior meniscus caused by the narrow medial space of the knee joint often encountered, which commonly leads to inaccurate surgical instrument operation, iatrogenic cartilage injury, excessive cutting of the meniscus and the failure of 
meniscus suture [3]. To address these problems, recent publications have reported local release of MCL through different methods to increase the gap of the posteromedial compartment, thereby improving the surgical visual field and the operating space under microscopy, and achieving satisfactory clinical results of medial meniscus surgery [2-6]. However, in these studies, the authors suggested MCL release without showing the specific release location, and some authors recommend that MCL release be performed with the assistance of a medial incision.

It is well known that the medial restrictive structure of the knee joint includes not only the MCL, but also the posterior oblique ligament (POL) and the capsule, collectively termed the posteromedial complex (PMC); Different parts of the PMC play different roles in knee flexion and extension [7]. During arthroscopic surgery of the posterior part of the medial meniscus in the half-extension position of the knee joint, the medial and posterior structures of the knee exert a restrictive effect on enlargement of the posteromedial space. Therefore, the structures being released should include the posterior part of the MCL, the POL and capsule. In this study, percutaneous pie-crusting release was performed at the medial and posterior part of the knee joint under valgus stress in the knee half-extension position, and the effect of release on the visual field of the posteromedial space of the knee joint was evaluated. The postoperative effect of release on the medial stability of the knee and the clinical efficacy of meniscal surgery were also evaluated.

\section{Methods}

1.1 Inclusion and exclusion criteria. The inclusion criteria were (1) age < 55 years, (2) pain on the medial side only, (3) varus angle $<5^{\circ}$, (4) injury of the posterior part of the medial meniscus, (5) arthroscopic exploration showed that the medial space was narrow in the semi-straight position of knee, the posterior aspect of the medial meniscus was not clearly visible, (6) need to perform release of the PMC of the knee, and (7) postoperative follow-up time of more than 1 year. The exclusion criteria were (1) degenerative injury of the meniscus, (2) Outerbridge grade of cartilage degeneration $\geq 3$ grade, (3) combined ligament or lateral meniscus injury, (5) previous knee operation, and (6) inflammatory arthritis. This study was approved by the Hospital Ethics Committee, and informed consents were obtained from all patients.

Based on aforementioned criteria, 60 patients with posterior injury of the medial meniscus from January 2016 to January 2018 were included in this study. The patients underwent pie-crusting release of the PMC under arthroscopy to improve the view field of the posteromedial space of the knee. Patients comprised 32 males and 28 females with an average age of $37.0 \pm 5.3$ years (16-55 years). The mean body mass index (BMI) was $25.0 \pm 2.1 \mathrm{~kg} / \mathrm{m}^{2}\left(21.5-31.4 \mathrm{~kg} / \mathrm{m}^{2}\right)$. Thirty-eight patients had injury to the left knee and 22 to the right knee. The mean time from injury to surgery was $35.5 \pm 2.1$ days (7-120 days). There were 26 cases with only posterior meniscus injury, and 34 patients with posterior plus body injury. Meniscoplasty were performed in 40 patients, and suturing was performed in 20 patients. According to the O'Connor classification of meniscus injury, there was a simple horizontal tear in 20 knees, a sagittal tear in eight cases, a longitudinal tear in 20 knees, and a complex tear in 12 knees. According to the Outerbridge classification of cartilage injury, 22 cases were grade 0,21 were grade $\rrbracket$, and 17 were grade $\otimes$ 
[8]. The clinical symptoms were locking, clicking, joint swelling and claudication. All operations were performed by a single surgeon.

1.2 Surgical technique. The load cell with capacity of $50 \mathrm{~kg}$ and accuracy of $0.01 \mathrm{~kg}$ (model L6D, ZEMIC Ltd., Hanzhong, Shaanxi, China) was mounted on the lateral block post of the operating table to monitor the amount of valgus stress that was applied to the patient's thigh during the operation. Under general or spinal anesthesia, the patient was positioned supine on the operating table with a tourniquet at the proximal thigh. Standard anterolateral (AL) and anteromedial (AM) approaches were used to examine meniscus, cartilage and ligaments. For patients with medial meniscus injury, the exposure of the posteromedial compartment was observed through the AL approach with the knee joint in a semi-straight position at 20 degrees. Valgus and external rotation stress of $11-\mathrm{kg}$ was applied to the leg by the surgeon using his body [9]. When the medial compartment was narrow, the posterior part of the medial meniscus was not clearly visible, and it was difficult to smoothly insert the blue forceps into the posterior space. Then controlled multi-point pie-crusting release of the MCL and POL were performed. The detailed procedures were as follows: (1) The probe hook was inserted into the medial joint space through AM approache, and the width of the posteromedial gap was evaluated with reference to whether the probe tip (4 mm height; Smith \& Nephew plc., London, UK) could pass through the narrow medial space vertically (Fig.1A). (2) At the near level of the medial joint line, percutaneous multi-point pie-crusting piercing of the posterior part of the MCL and the POL using an 18-G venous needle was carried out. It is appropriate to sense a "slight tear" when piercing the aforementioned tissues (Fig.1B). (3) Under 11-kg valgus stress, multi-point release stopped when the posteromedial space was suddenly opened. At this time, the posterior part of the meniscus could be clearly viewed, and the probe tip could easily pass through the narrow space in the vertical orientation (Fig.1C). (4) When the posteromedial space was opened satisfactorily, meniscoplasty or suture could be performed.

1.3 Postoperative management. After recovery from anesthesia, isometric contraction of the quadriceps femoris and straight leg elevation training were performed. Patients who underwent meniscoplasty were recommended to wear an short knee brace for 4 weeks to prevent further injury of the MCL, and were allowed to do full-weight bearing and full range of motion exercise two days after operation. Patients who underwent meniscal suture were encouraged to practice ambulation without weight-bearing on the affected limbs under the protection of a adjustable brace and crutch. Four weeks after operation, patients carried out partial weight-bearing activities, then gradually the brace was removed and full weight-bearing activities were allowed six weeks after operation, with active knee flexion more than $120^{\circ}$. Stationary cycling and moderate intensity running were allowed 3 months after surgery, and full return to sport was permitted 6 months later. 
1.4 Arthroscopic measurements of the joint space width (JSW). The intraoperative width of the medial space before and after release of the PMC was measured as follows. The probe hook was inserted through the AM approach, and its vertical tip was placed against the lowest point of the femoral condyle cartilage at the narrow space. Before release, the vertical tip $(4 \mathrm{~mm})$ often formed an acute angle with the articular surface; The width of the narrow space (h1) can be calculated by the sine formula ( $\mathrm{h} 1=4 \mathrm{~mm} \times$ $\sin A$ ), where $A$ is the angle between the vertical tip and the articular surface (Fig.2a). After release, the medial space was enlarged, and the vertical tip could pass vertically through the narrow space, and the opening width of the medial gap ( $\mathrm{h} 2$ ) can be calculated by the formula ( $\mathrm{h} 2=4 / \mathrm{H} 1 \times \mathrm{H} 2+4 \mathrm{~mm}$ ), where $\mathrm{H} 1$ is the magnified measured height of the vertical tip of the hook under microscopy (greater than 4 $\mathrm{mm}$ ), and $\mathrm{H} 2$ is the magnified measured height between the tip of the hook and the lowest point of the femoral condyle under microscopy (Fig.2b). h1-h2 is the extra opening width. The height of $\mathrm{H} 1$ and $\mathrm{H} 2$ was measured with a digital calliper with accuracy to $0.1 \mathrm{~mm}$.

1.5 Radiographic measurements of the JSW. The identical load cell was mounted on the stress radiography device to monitor the amount of valgus stress that was applied to the patient's thigh during performing X-ray films. With the patient in supine position and the knee in a semi-straight position at 20 degrees, an anteroposterior (AP) knee radiograph with 11-kg valgus stress of the lower leg was taken with the X-ray beam centred in the joint line and the tube distance of 1 metre from the cassette. JSW was measured using a picture archiving and communication system (PACS) as follows with modification [9]. On an AP X-ray film, a line was drawn to connect the subchondral bone of the medial and lateral tibial condyle. From this line, a perpendicular line was drawn to the most distal point of the medial femoral condyle. The distance of this vertical line recorded as JSW and measured using PACS software (PACS, PI View STAR, version 5025; Infinitt, Seoul, Korea) (Fig. 3).

1.6 MRI analysis of the meniscus and MCL healing. All MRI examinations of the knee was performed using a 3.0-T system before and after operation. A special superficial sagittal section was taken to evaluate the localization of the PMC injury. In coronal sections, the localization of the injury was recorded with reference to the medial meniscus [9]. Based on MRI signal grade, the meniscus was considered completely healed if there was no fluid signal within it, incompletely healed if an intrameniscal signal approached only one articular surface, and unhealed if the signal hyperintensity extended from one articular surface to the other [10].

1.7 Clinical evaluation. To evaluate the effect of arthroscopic pie-crusting release during operation, the width of the medial space before and after release was measured; the improvement of the visual field and occurrence of iatrogenic cartilage injury were also observed. During the follow-up, the medial stability of the knee joint was evaluated by radiographic measurements of the JSW preoperatively and in the 
postoperative 1st week and 3rd months. Healing of the MCL and sutured meniscus was evaluated by MRI. VAS [11], Lysholm score [12], Tegner score [13], and IKDC score [14] were used to evaluate knee joint functions.

1.8 Statistical analysis. The measurement data are expressed as mean \pm standard deviation. Student's paired t-test was used to test the significance of differences, and $\mathrm{P}<0.05$ denoted a significant difference. All statistical analyses were performed with SPSS 15.0 (SPSS Inc., Chicago, IL, USA).

\section{Results}

After pie-crusting release of the PMC of the knee joint, the intraoperative posteromedial gap was obviously increased. The width of the medial space, as calculated from the arthroscopic photographs and formula, was $2.5 \pm 0.5 \mathrm{~mm}(1.9-4 \mathrm{~mm})$ before release and $5.7 \pm 0.5 \mathrm{~mm}(4-7 \mathrm{~mm})$ after release, and the difference was statistically significant $(p<0.01$ ) (Fig. 4a). The extra increased width was $3.2 \pm 0.6 \mathrm{~mm}$ (2-4.4 mm). The location and morphology of the injuried meniscus were clearly visible, and no obvious iatrogenic cartilage injury occurred during operation.

The patients were followed up for $21.93 \pm 7.04$ months (12-36 months). At the final follow-up, The JSW, as calculated from the X-ray radiographs under valgus stress, was $5.9 \pm 0.8 \mathrm{~mm}(4-7.5 \mathrm{~mm})$ preoperatively, $9.2 \pm 1.1 \mathrm{~mm}(7-11 \mathrm{~mm})$ in the postoperative $1 \mathrm{st}$ week, and $6.1 \pm 0.9 \mathrm{~mm}(4.2-7.6 \mathrm{~mm})$ in the postoperative $3 \mathrm{rd}$ months, the difference between preoperative and 1st week was statistically significant $(p<0.01)$; However, there was no significant difference between the preoperative and postoperative 3rd month ( $p>0.05$ ) (Fig. 3, Fig. 4b).

At the final follow-up, of the 20 patients with meniscus suture, MRI showed complete meniscus healing in 15 patients and two-grade abnormal signals in five patients who had no obvious tenderness in the medial space. For all patients, VAS, Lysholm, IKDC and Tegner scores were $1.80 \pm 0.51$ (1-3), $80.08 \pm 3.74$ (70$85), 82.17 \pm 4.64(75-90)$ and $5.48 \pm 0.59(4-7)$, respectively, showing significant differences compared with the preoperative scores [5.57 $\pm 0.69(4-7), 48.17 \pm 4.22(40-55), 51.42 \pm 4.02(45-55)$ and $3.20 \pm$ $0.68(2-4)$, respectively) $(P<0.01)$ (See Table 1). Typical cases were seen Fig. 5 and Fig. 6.

\section{Discussion}

For posterior injury of the medial meniscus with clinical symptoms, arthroscopic minimally-invasive surgery is often required. The difficulty during operation is that the posteromedial compartment is difficult to expose clearly because of the narrow joint gap, which results in a limited visual field of the posterior meniscus and a lack of space for manipulation of the surgical instruments under arthroscopy [2-6]. Consequently, it is important for arthroscopic surgeons to seek other methods to enlarge the posteromedial space of the knee joint, one of which is the use of the pie-crusting release technique to release the medial structure of the knee under arthroscopy in order to increase the medial joint gap, and 
ultimately improve the intraoperative visual field and operation space [15-17]. In total knee arthroplasty, in order to achieve the lateral and medial balance of the extension and flexion gap, Mihalko et al. performed pie-crusting acupuncture release of the $\mathrm{MCL}$, and the joint space during flexion and extension was simultaneously increased and balanced [13]. Bellemans et al. used this technique to release the MCL with a 19-G needle in the vicinity of the knee joint line, which produced a mean $2.4 \mathrm{~mm}$ increase of the medial joint space [15]. It has been reported that application of this technique to the opening of the medial space of the knee joint under arthroscopy, whether through an open skin incision or percutaneous acupuncture for $M C L$ release, improves the medial visual field $[2,6,18-20]$. In this study, the pie-crusting technique was used to release the PMC of the knee joint, and a satisfactory visual field of the posterior joint space was obtained under valgus stress of the knee in a half-extension position, in which not only the posterior shape of the meniscus was fully revealed, but also it was possible to insert the surgical instruments into the posterior space accurately and smoothly, and no further iatrogenic cartilage injury occurred.

For increasing the width of the posteromedial space of the knee joint under arthroscopy, it is not necessary to release the whole medial stable structure. For arthroscopic treatment of medial meniscus injury in this study, the position of the lower extremity is usually near extension, in which the main structure restricting knee valgus is the posteromedial stable structure. Reports in the literature have shown that the posteromedial stable structure of the knee joint mainly consists of the posterior part of the MCL, the POL and the posteromedial capsule [21]. The anterior part of the MCL maintains tension and restricts excessive knee valgus in the flexion position. The posterior fiber of the MCL maintains tension in the extension position, restricting knee valgus in the knee half-extension position ( 20 degrees of flexion). The POL is the thickening part of the posteromedial side of the articular capsule, the proximal end of the POL is attached to the adductor tubercle of the femur or between the adductor tubercle and the medial femoral condyle, then expands distally and posteriorly and attaches to the posterior side of the tibia, and its main central or tibial bundle attaches to the posteromedial side of the tibial plateau near to the joint surface, preventing knee valgus in a half-extension position [21]. It can be seen from this analysis that the posterior part of the MCL and the POL are the main tissue structures which restrict the opening of the posteromedial space in the half-extension position under knee valgus. We performed multi-point percutaneous acupuncture for the posterior part of the $\mathrm{MCL}, \mathrm{POL}$ and articular capsule using an 18-G needle at the posterior $1 / 3$ region of the knee joint, satisfactory posterior space exposure was obtained under valgus stress.

The clinical results have shown that pie-crusting release of the PMC can obviously improve the visual field of the posteromedial space of the knee joint. However, a question arising from this technique is whether the release can result in the risk of medial instability of the knee after surgery. Through imaging study in the literature about the changes in the medial gap after MCL release, it was found that the release caused a partial tear of the MCL, and the medial space was $2 \mathrm{~mm}, 0.9 \mathrm{~mm}$ and $0.1 \mathrm{~mm}$ at 1 week, 1 month and 3 months after operation, respectively [20]. Another study showed that the MCL injury was rated $I^{\circ}$ in the valgus position with $20^{\circ}$ flexion immediately after operation but negative at 6 weeks after operation, and there was no subjective feeling of medial instability of the knee during follow-up [6]. A 
similar result was also found in our study, the patients had no subjective or objective medial instability of the knee joint at 3 months after operation, the joint space width from X-ray radiographs was $6.13 \pm$ $0.89 \mathrm{~mm}$, showing no significant difference compared with preoperative $5.97 \pm 0.89 \mathrm{~mm}$, indicating the healing of the injured PMC. The main factors related to knee stability after pie-crusting release of the PMC were as follows: (1) In this technique, only the posterior part of the MCL and POL were pierced, the anterior part of the MCL was intact. (2) The extra intraoperative opening width of the medial space after release was mean $3.19 \mathrm{~mm}$, which only maintained relaxation of the posteromedial stable structures within the range of $I^{\circ}$ injury ( $<5 \mathrm{~mm}$ opening on the medial side) [9]. (3) Patients were required to wear brace for AT LEAST 4 weeks after operation, which ensured the healing of the sutured meniscus and released posteromedial stable structure, thus avoiding the occurrence of iatrogenic knee instability.

The following surgical tips for arthroscopic pie-crusting release of the PMC should be noted. (1) While performing pie-crusting release, the knee joint should be maintained in slight flexion and abduction to ensure a certain tension of the PMC[6]. (2) Percutaneous puncture should be performed $1.0 \mathrm{~cm}$ above and below the joint line level so as to avoid possible injury to the saphenous nerve and great saphenous vein. (3) The puncture should be carried out from the posterior part to the middle part of the PMC row by row until feeling a "slight tear". (4) The release standard should be that the probe tip could easily pass through the narrow joint space in the vertical orientation, or the blue forceps could easily enter the posterior space. (5) The knee joint should be fixed with an brace after operation to promote healing of the released MCL.

\section{Conclusions}

Arthroscopic pie-crusting release of the PMC can effectively increase the posteromedial space and improve the visual field of the knee joint under arthroscopy, and this technique do neither produce any residual valgus instability of the knee nor affect clinical outcome at the final follow-up.

\section{Abbreviations}

MCL

Medial collateral ligament; PMC:Posteromedial complex; POL:Posterior oblique ligament; JSW:Joint space width; MRI:Magnetic resonance imaging; VAS:Visual analogue scale; IKDC:International Knee Documentation Committee; BMI:Body mass index; AL:Anterolatera; AM:Anteromedial

\section{Declarations}

\section{Ethics approval and consent to participate}

The study was approved by the Ethics Committee of Henan Luoyang Orthopedic-Traumatological Hospital (Henan Orthopedic Hospital). Informed written consent was obtained from each participant, and children (under 16 years old) from their parent. 


\section{Consent for publication}

Written informed consent was obtained from the patients for publication of this report and any accompanying images.

\section{Availability of data and materials}

The datasets used and/or analysed during the current study are available from the corresponding author on reasonable request.

\section{Competing interests}

The authors declare that they have no competing interests.

\section{Funding}

No specific funding was received from any funding bodies

\section{Authors' contributions}

HLT designed this study and approved the final version of the manuscript; XH and PZW wrote the manuscript; JYY and XW collected the clinical data. All the authors read and approved the final manuscript.

\section{Acknowledgements}

Not applicable.

\section{Author details}

${ }^{1}$ Henan Luoyang Orthopedic-Traumatological Hospital (Henan Orthopedic Hospital), No.80 Qingming southern Road, Luoyang 471002, China. ${ }^{2}$ Hunan University of Chinese Traditional Medicine, Hunan, Changsa 410208, China.

\section{References}

1.Henry S, Mascarenhas R, Kowalchuk D, Forsythe B, Irrgang JJ, Harner CD. Medial meniscus tear morphology and chondral degeneration of the knee:is there a relationship? Arthroscopy, 2012;28(8):112434. 
Liu X, Feng H, Zhang H.The application of Pie-crusting technique of superficial medial collateral ligament under arthroscopy in repairing medial meniscus tears. Chinese Journal of Sports Medicine.2017;36(1):58.

Atoun E, Debbi R, Lubovsky O, Weiler, A, Debbi, E, Rath, E.Arthroscopic transportal deep medial collateral ligament pie-crusting release. Arthrosc Tech.2013; 2(1):e41-3.

Park YS, Moon HK, Koh YG, Kim YC, Sim DS, Jo SB, Kwon SK. Arthroscopic pullout repair of posterior root tear of the medial meniscus:the anterior approach using medial collateral ligament pie-crusting release. Knee Surg Sports Traumatol Arthrosc. 2011;19(8):1334-6.

5.Roussignol X, Gauthe R, Rahali S, Mandereau C, Courage O, Duparc F. Opening the medial tibiofemoral compartment by pie-crusting the superficial medial collateral ligament at its tibial insertion: a cadaver study. Orthop Traumatol Surg Res. 2015;101(5):529-33.

6.Zhu WH, Tang Q, Liao LL, Li D, Yang Y, Chen Y. Application of pie-crusting the medial collateral ligament release in arthroscopic surgery for posterior horn of medial meniscus in knee join. J Cent South Univ (Med Sci).2017;42(9):1053-7.

Hosseini A, Qi W, Tsai TY, Liu YJ, Rubash H, Li GA.In vivo length change patterns of the medial and lateral collateral ligaments along the flexion path of the knee. Knee Surg Sports Traumatol Arthrosc.2015;23(10):3055-61.

8.Outerbridge RE. The etiology of chondromalacia patellae. J Bone Joint Surg Br.1961;43-B:752-7.

Onur FO, Ozsoy MH, Ozdemir HM, Yigit H, Cavusoglu AT, Lobenhoffer P. Percutaneous medial collateral ligament release in arthroscopic medial meniscectomy in tight knees. Knee Surg Sports Traumatol Arthrosc.2013;21(7):1540-5.

10.Yoon KH, Park JY, Kwon YB, Lee YJ, Kim EJ, Kim SG. Inside-out repair of the meniscus in concomitant anterior cruciate ligament reconstruction: absorbable versus nonabsorbable sutures.Arthroscopy. 2020;13;DOI:10.1016/j.arthro.2019.08.045.

11.Aitken RBC. Measurement of feelings using visual analogue scales. Proc R Soc Med.1969;62(10):98993.

12.Lysholm J, Gillquist J. Evaluation of knee ligament surgery results with special emphasis on use of a scoring scale. Am J Sports Med. 1982;10(3):150-4.

13.Tegner Y, Lyshom J. Rating systems in the evaluation of knee ligament injuries. Clin Orthop Relat Res.1985;98:43-9.

14. Hefti F, Müller W, Jakob RP, Stäubli HU. Evaluation of knee ligament injuries with the IKDC form. Knee Surg Sports Traumatol Arthrosc.1993; 1(3-4):226-34. 
Mihalko WM, Woodard EL, Hebert CT, Crockarell JR, Williams JL. Biomechanical validation of medial piecrusting for soft tissue balancing in knee arthroplasty. J Arthroplasty.2015;30(2):296-9.

Kwak DS, In Y, Kim TK, Cho HS, Koh IJ. The pie-crusting technique using a blade knife for medial collateral ligament release is unreliable in varus total knee arthroplasty. Knee Surg Sports Traumatol Arthrosc.2016;24(1):188-94.

17.Bellemans J, Vandenneucker $\mathrm{H}$, Van Lauwe J, Victor $\mathrm{J}$. A new surgical technique for medial collateral ligament balancing: multiple needle puncturing.J Arthroplasty.2010; 25(7):1151-6.

18.Claret G, Montañana J, Rios J, Ruiz-Ibán MA, Popescu D, Núñez M, Lozano L, Combalia A, Sastre S. The effect of percutaneous release of the medial collateral ligament in arthroscopic medial meniscectomy on functional outcome. Knee.2016; 23(2):251-5.

19.Todor A, Caterev S, Nistor DV. Outside-in deep medial collateral ligament release during arthroscopic medial meniscus surgery.Arthrosc Tech.2016;5(4): e781-e5.

20.Fakioglu O, Ozsoy MH, Ozdemir HM, Yigit H, Cavusoglu AT, Lobenhoffer P. Percutaneous medial collateral ligament release in arthroscopic medial meniscectomy in tight knees. Knee Surg Sports Traumatol Arthrosc.2013;21(7):1540-5.

21.Tan HF, Bai LH. Anatomy and biomechanical research progress of knee posteromedial corner. Chin J Repar Reconstr Surg.2019; 23(9):1058-61.

\section{Table 1}

Table 1 Clinical assessment results $(n=60$, Mean \pm SD)

\begin{tabular}{lccc}
\hline & Preoperation & Final follow-up & $P$ value \\
\hline VAS & $5.57 \pm 0.69$ & $1.80 \pm 0.51$ & 0.00 \\
Lysholm & $48.17 \pm 4.18$ & $80.08 \pm 3.71$ & 0.00 \\
\hline IKDC & $51.42 \pm 3.99$ & $82.17 \pm 4.59$ & 0.00 \\
\hline Tegner & $3.20 \pm 0.68$ & $5.48 \pm 0.59$ & 0.00 \\
\hline
\end{tabular}

\section{Figures}




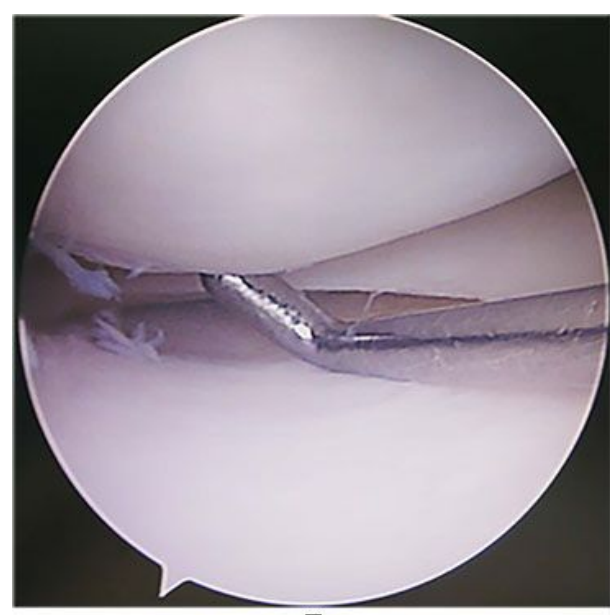

a

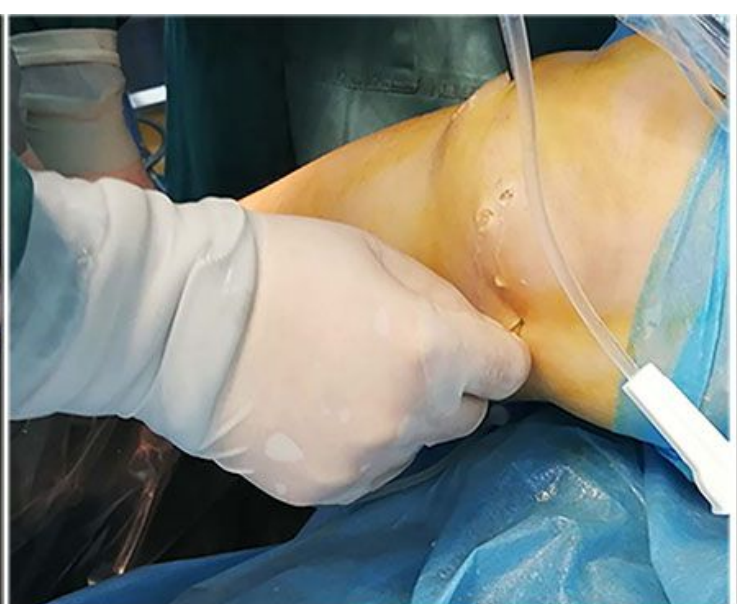

b

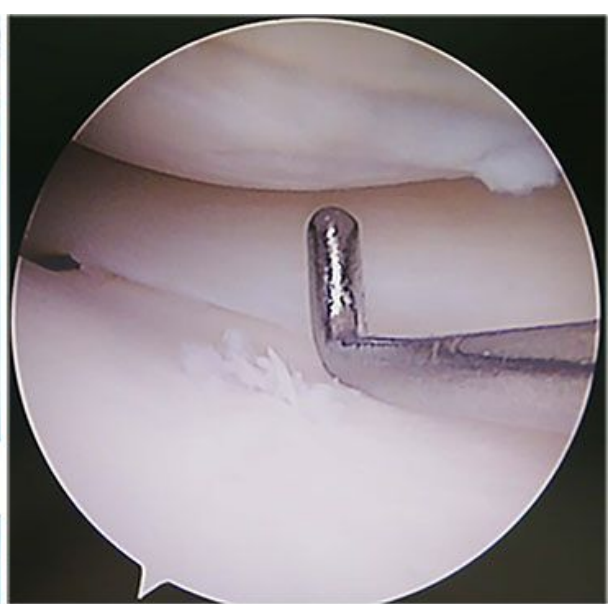

C

Figure 1

Arthroscopic pie-crusting release of the PMC. a With the knee joint in the valgus position, the probe did not vertically pass through the narrow medial space. $b$ Multi-point pie-crusting release of the PMC with an 18-G needle. c After release, the probe passed vertically through the medial space, and the visual field was improved 


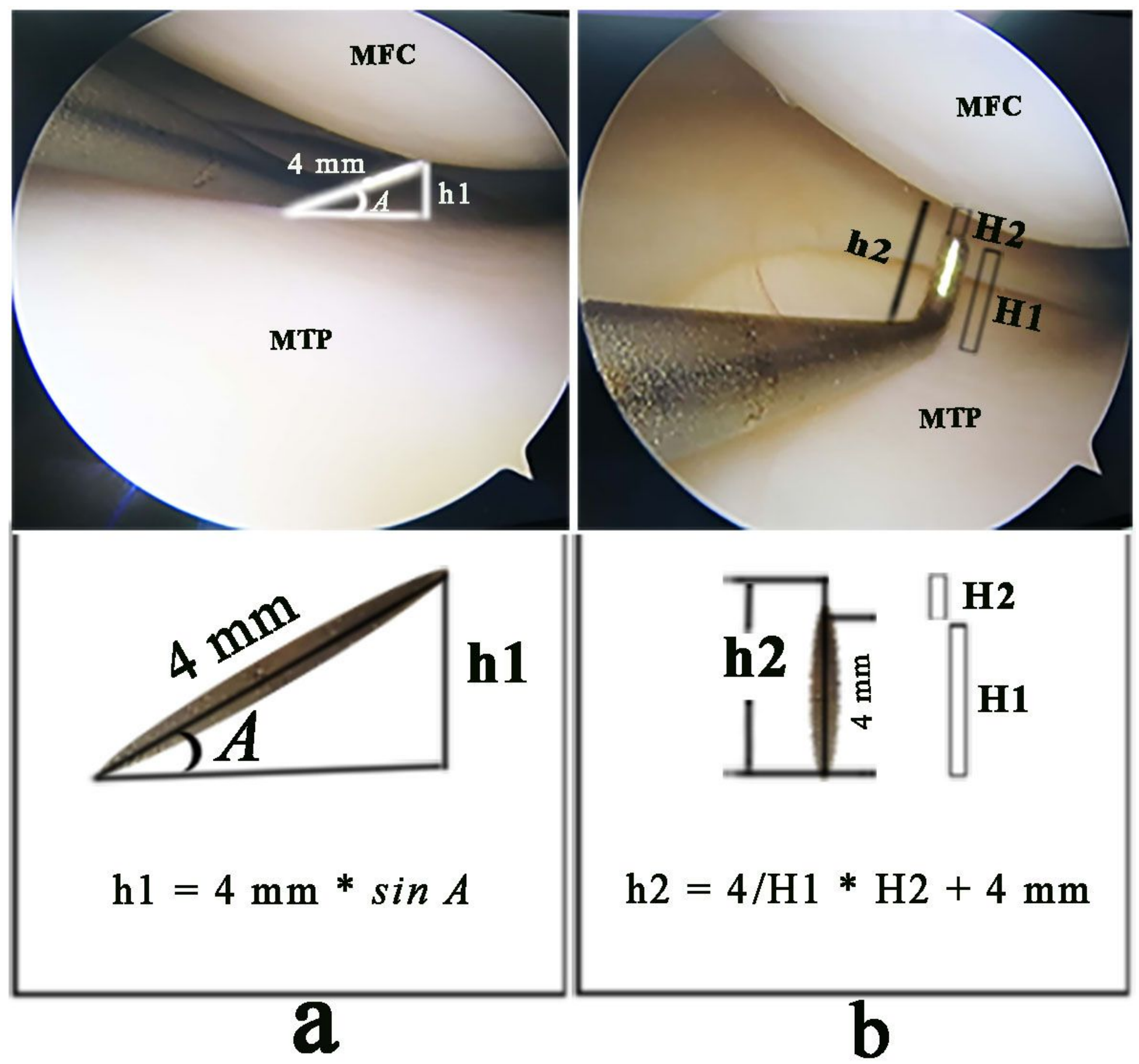

Figure 2

Arthroscopic measurements of the joint space width (JSW). a Before pie-scrusting release. b After Piescrusting release. MFC, medial femoral condyle. MTP, medial tibial plateau 


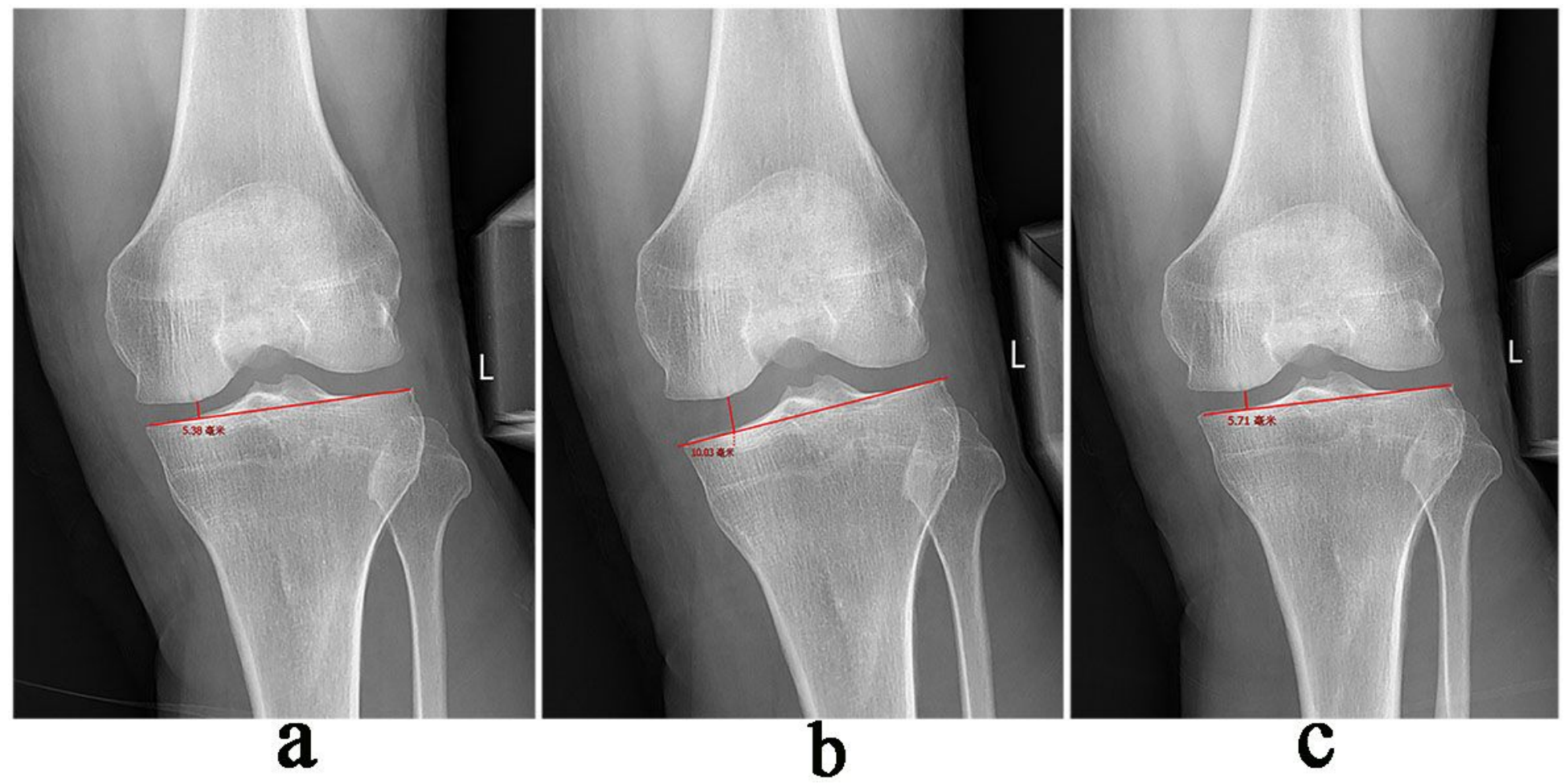

\section{Figure 3}

Radiographic measurement of JSW under valgus stress radiograph. The distance of JSW (perpendicular line) was measured using PACS software. a The JSW was $5.38 \mathrm{~mm}$ preoperatively. b $10.03 \mathrm{~mm}$ in the postoperative 1st week. c $5.71 \mathrm{~mm}$ in the postoperative 3rd months 

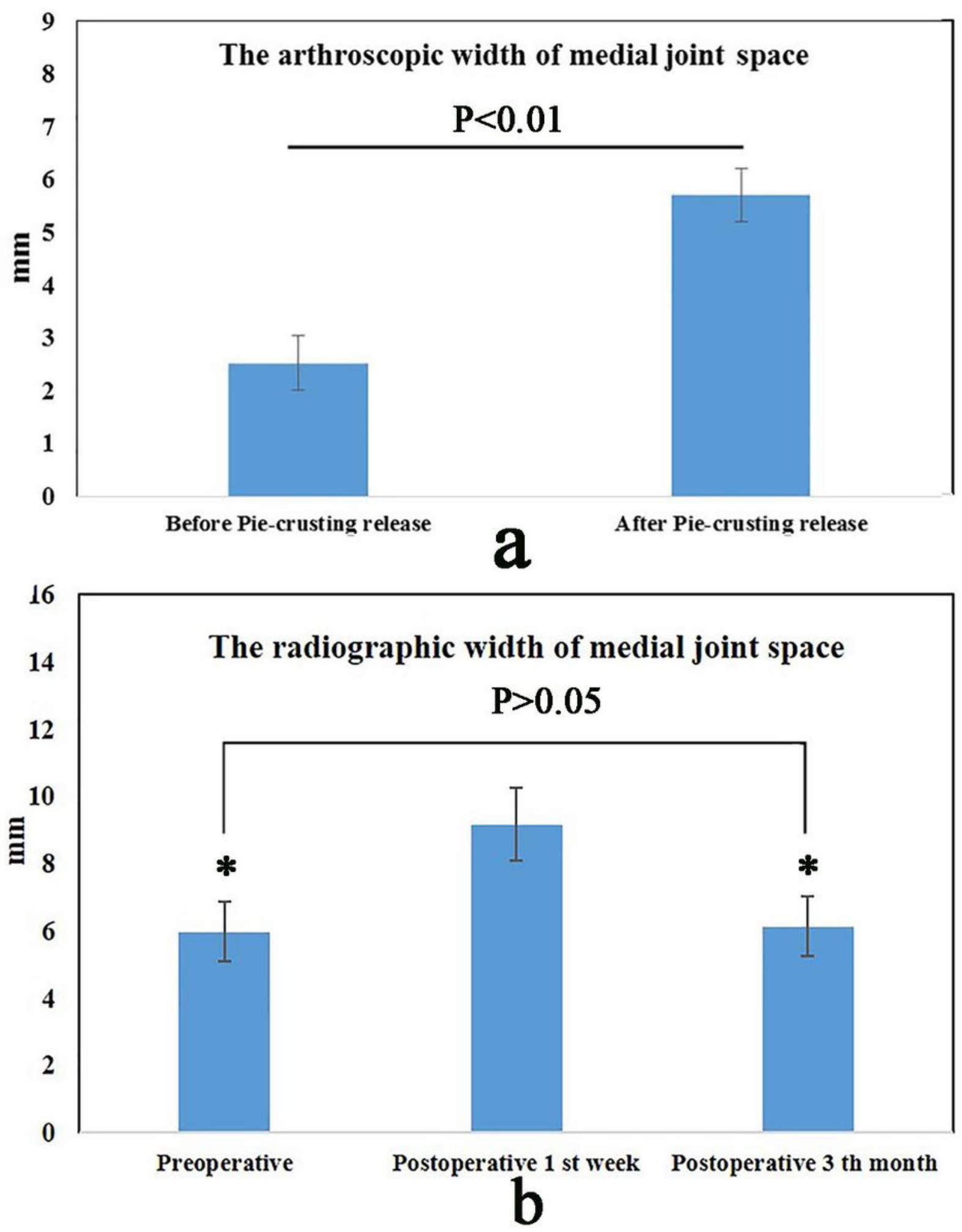

Figure 4

Measurement of medial JSW of the knee joint. a Arthroscopic measurement of JSW. b Radiographic measurement of JSW, compared with the value of postoperative 1 st week. ${ }^{*} p<0.01$ 


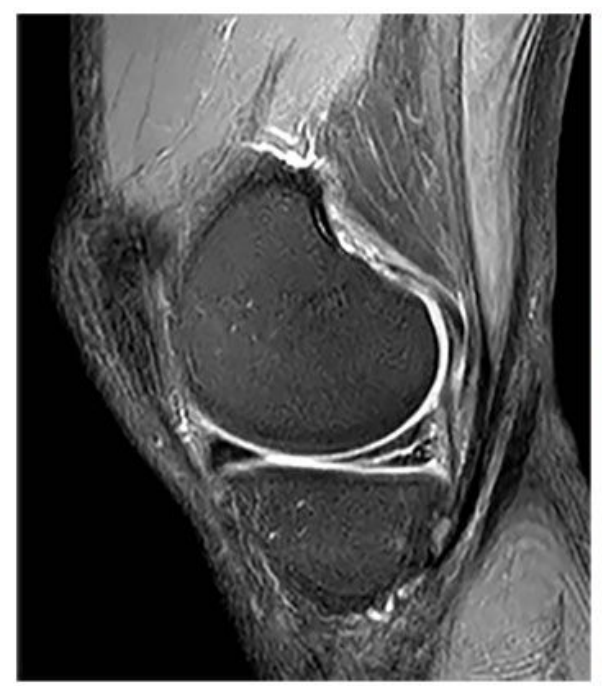

a
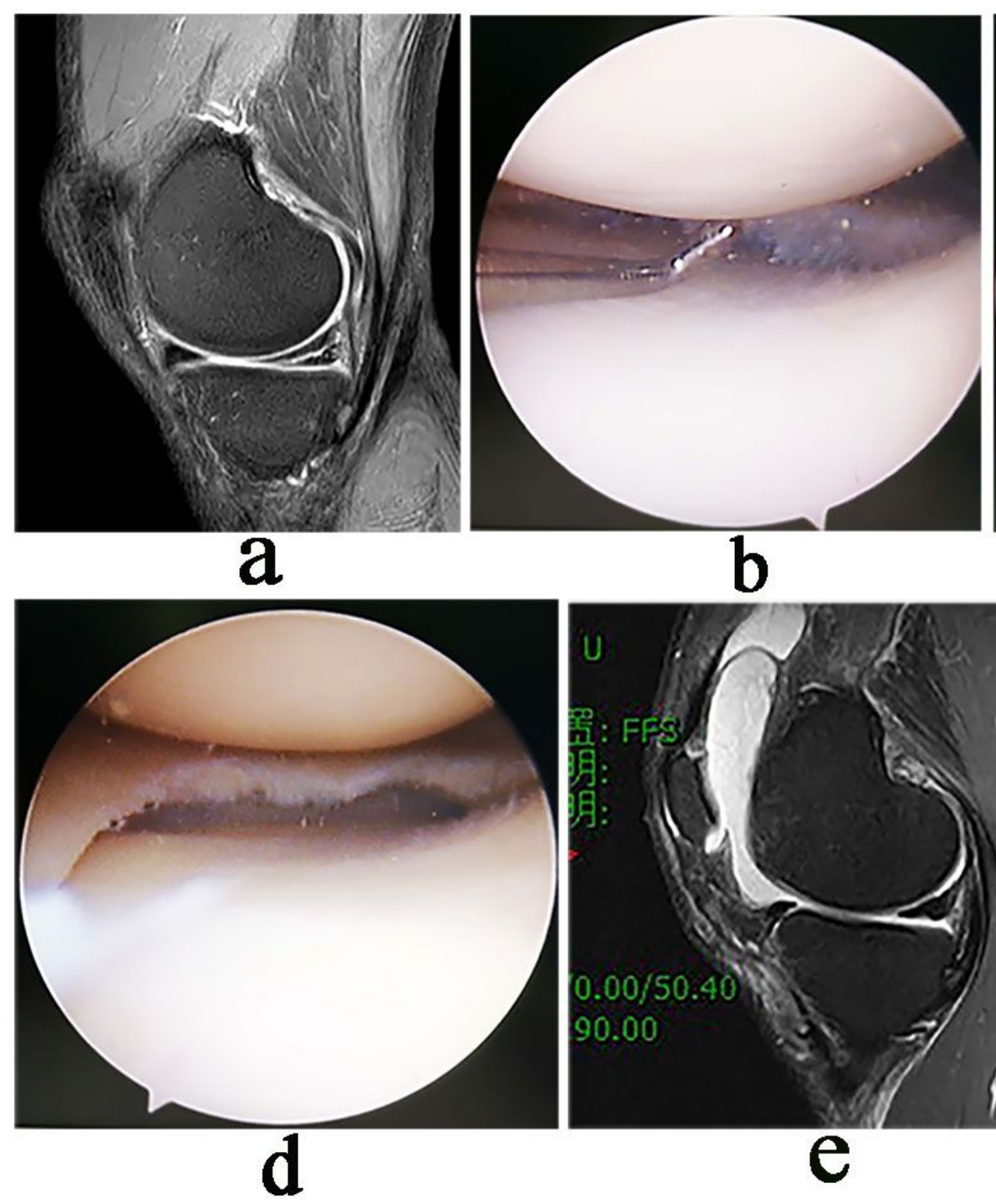

b

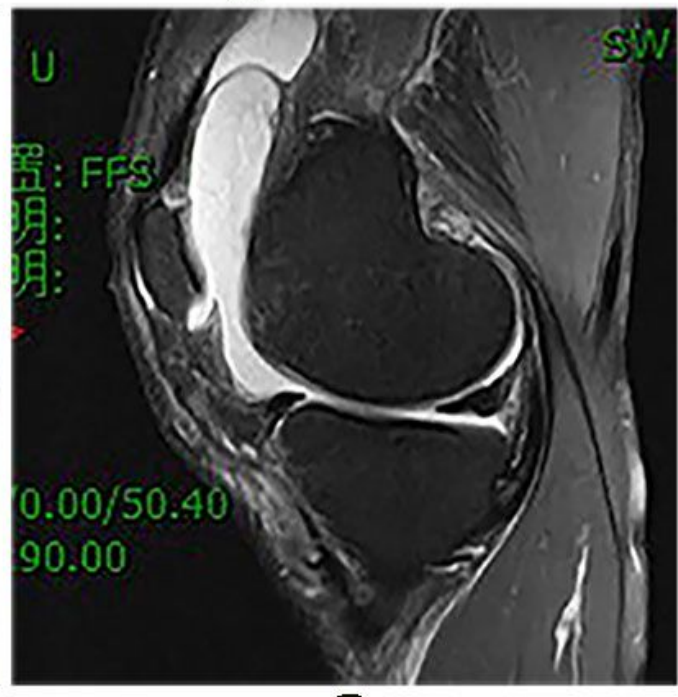

e

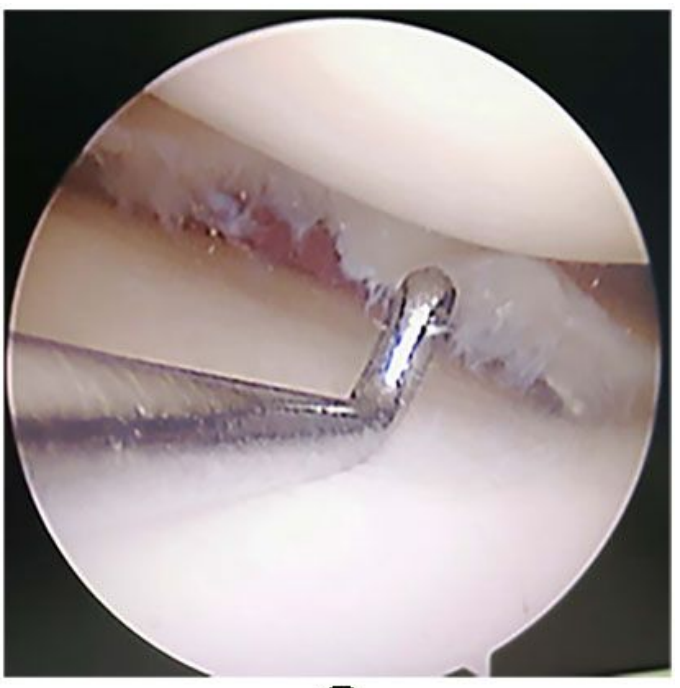

C

\section{Figure 5}

Arthroscopic pie-crusting release of PMC for meniscoplasty. a Preoperative MRI showed a horizontal tear of the medial meniscus. b Arthroscopic visual field of the posteromedial space was not clear, and the probe tip could not vertically pass through the narrow gap. c After release, the probe tip could pass vertically through the narrow gap, and the visual field was improved. $d$ Meniscoplasty of the posterior meniscus was performed without iatrogenic cartilage injury. e After 18 months follow-up, the shape of the posterior meniscus was satisfactory 


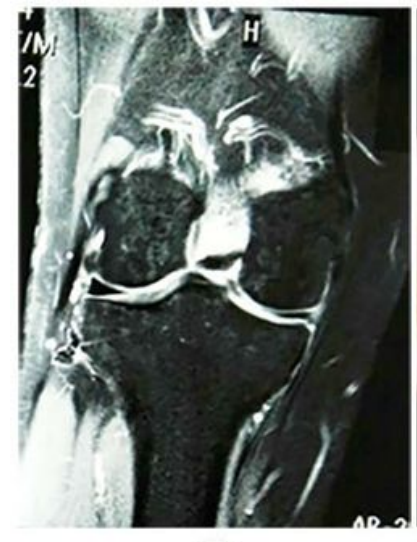

a

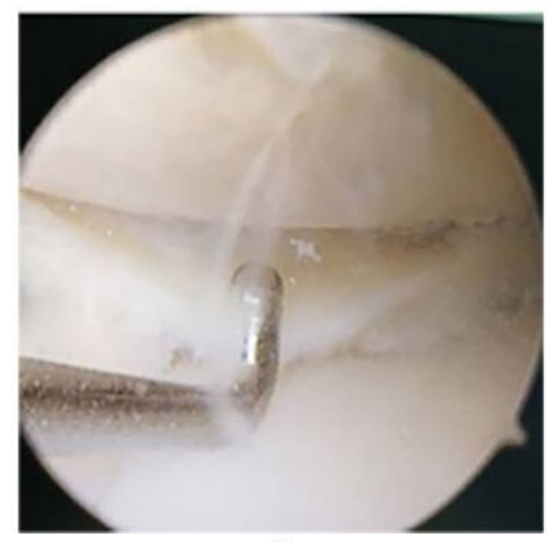

e

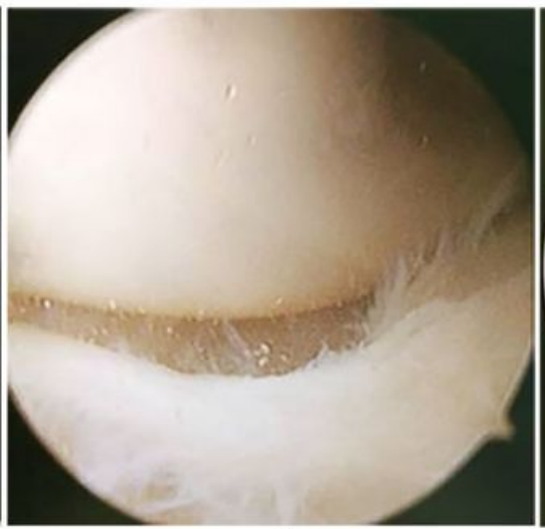

b

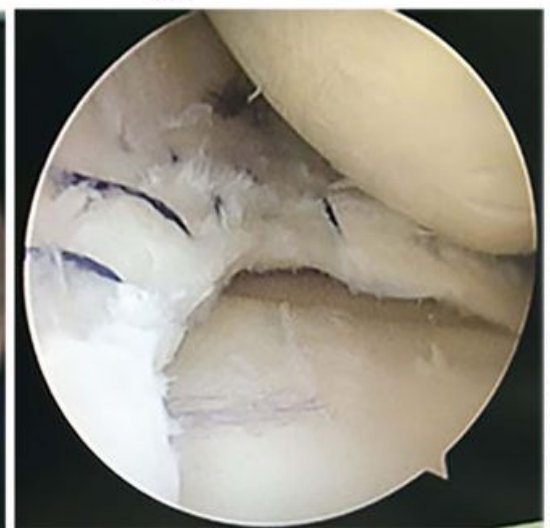

f

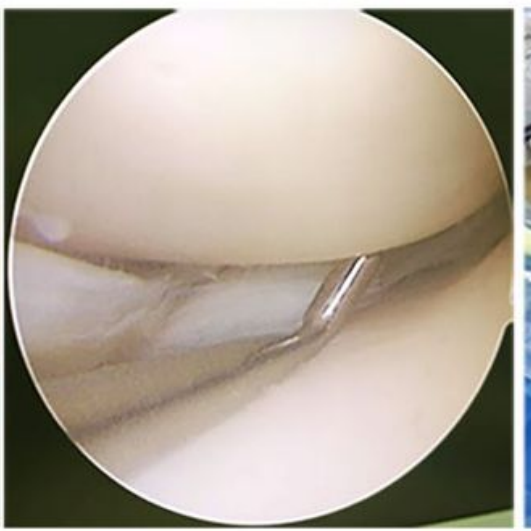

C

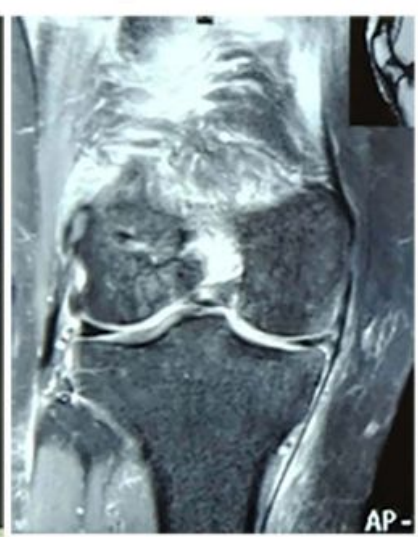

g

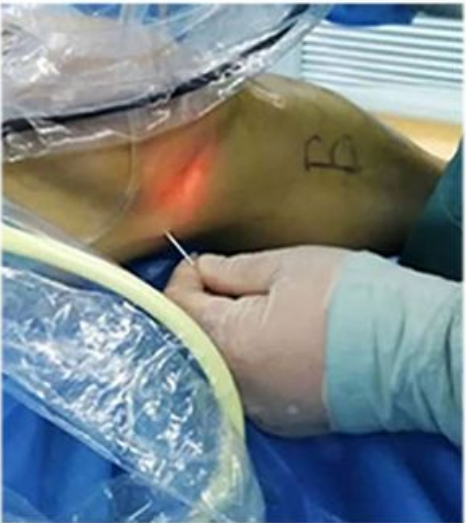

d

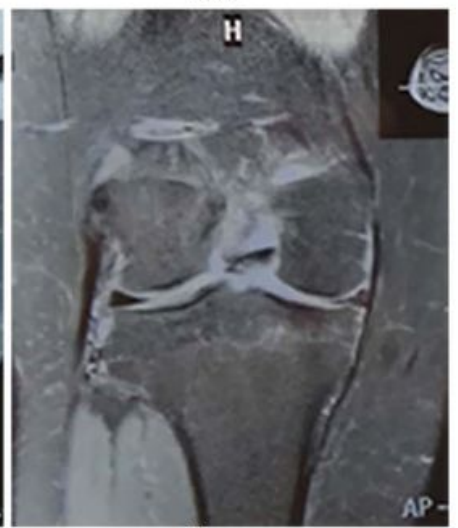

h

\section{Figure 6}

Arthroscopic pie-crusting release of PMC for meniscus suture. a Preoperative MRI showed a buckethandle tear of the medial meniscus. b Displacement of the injured meniscus under arthroscopy. c The injured meniscus was reduced, but the probe tip could not pass vertically through the medial gap. $d$ Multipoint release of the PMC. e After release, the probe tip could pass vertically through the narrow gap. $f$ The meniscus was sutured, with no iatrogenic cartilage injury. g Postoperative MRI showed normal meniscus shape and mild iatrogenic injury of MCL. h After 18 months follow-up, MRI showed meniscus and MCL healing

\section{Supplementary Files}

This is a list of supplementary files associated with this preprint. Click to download.

- Clinicaldata.xlsx 